\title{
Erratum: Refined gluino and squark pole masses beyond leading order [Phys. Rev. D 74, 075009 (2006)]
}

\author{
Stephen P. Martin
}

Q (Received 11 December 2018; published 27 December 2018)

DOI: 10.1103/PhysRevD.98.119901

In Eq. (A11), $C_{a}(G)$ should be $C_{a}(A)$.

In Eqs. (A17) and (A18), there were several incorrect coefficients. These equations should read:

$$
\begin{aligned}
x^{(2)}= & h_{a}^{2} C_{a}(F)\left\{C_{a}(A)\left[-1111 / 24+4 \pi^{2} / 3-4 \pi^{2} \ln 2+6 \zeta_{3}\right]+S_{a}^{L}\left[71+8 \pi^{2}\right] / 12\right. \\
& \left.+S_{a}^{H}\left[143-16 \pi^{2}\right] / 12\right\}+h_{a} h_{b} C_{a}(F) C_{b}(F)\left[8 \pi^{2} \ln 2-5 \pi^{2}-12 \zeta_{3}+7 / 8\right],
\end{aligned}
$$

and

$$
\begin{aligned}
x^{(3)}= & 64 h_{a} C_{a}(F)\left[h_{b} h_{c} C_{b}(F) C_{c}(F) d_{1}^{(3)}+h_{a} h_{b}\left\{C_{a}(A) C_{b}(F) d_{2}^{(3)}+S_{a b}^{L} d_{4 A}^{(3)} / 2+S_{a}^{L} C_{b}(F) d_{4 B}^{(3)} / 2\right.\right. \\
& \left.+S_{a b}^{H} d_{5}^{(3)} / 2\right\}+h_{a}^{2}\left\{\left[C_{a}(A)\right]^{2} d_{3}^{(3)}+C_{a}(A) S_{a}^{L} d_{6}^{(3)} / 2+C_{a}(A) S_{a}^{H} d_{7}^{(3)} / 2+S_{a}^{L} S_{a}^{H} d_{8}^{(3)} / 4\right. \\
& \left.\left.+\left[S_{a}^{H}\right]^{2} d_{9}^{(3)} / 4+\left[S_{a}^{L}\right]^{2} d_{10}^{(3)} / 4\right\}\right] .
\end{aligned}
$$

